\title{
Ethyl pyruvate prevents from chronic cerebral hypoperfusion via preserving cognitive function and decreasing oxidative stress, caspase 3 activation and IL-1 $\beta$ level
}

\author{
Sayan Ozacmak H, Ozacmak VH, Turan I \\ Bulent Ecevit University Faculty of Medicine, Department of Physiology, Zonguldak, Turkey. hsayan@yahoo.com
}

\begin{abstract}
BACKGROUND: One of the important risk factors for dementia is chronic cerebral hypoperfusion $(\mathrm{CCH})$ especially in patients with cerebrovascular disease.

OBJECTIVES: In the present study, using rat model of bilateral common carotid artery occlusion, the possible protective effects of ethyl pyruvate (EP) have been explored in terms of memory impairment, oxidative stress, and levels of caspase-3, Na-K ATPase, and IL- $1 \beta$.

METHODS: Rats were treated with EP $(50 \mathrm{mg} / \mathrm{kg}$, i.p) for 4 weeks. Cognitive function was evaluated by Morris Water Maze (MWM). Both levels of caspase- 3 and Na-K ATPase in tissue, IL-1 $\beta$ in plasma were measured by ELISA method. Status of oxidative stress in brain was assessed by the measurements of the tissue malondialdehyde (MDA) and reduced glutathione (GSH) contents.

RESULTS: Results showed that $\mathrm{CCH}$ caused a striking impairment of spatial working memory, accompanied with increased levels of MDA and IL-1 $\beta$ as well as caspase 3 level. The treatment with EP, however, significantly improved the memory impairment. Moreover, the treatment also provided beneficial effects on the disturbances of caspase $3, \mathrm{IL}-1 \beta$ and MDA.

CONCLUSION: This study strongly imply that the EP administration can alleviate the memory impairment observed due to $\mathrm{CCH}$. The protection provided by EP may result from inhibition of inflammatory response, apoptotic processes and oxidative stress (Fig. 3, Ref. 58). Text in PDF www.elis.sk.

KEY WORDS: ethyl pyruvate, oxidative stress, chronic cerebral hypoperfusion, caspase 3, memory impairment.
\end{abstract}

\section{Introduction}

Chronic cerebral hypoperfusion $(\mathrm{CCH})$ is a common pathophysiological state, which is identified as a long-term, mild ischemic brain insult and is involved with the initiation and progression of vascular dementia (VD) and Alzheimer's disease (AD) $(1,2)$.

In order to explore $\mathrm{CCH}$ and $\mathrm{CCH}$-related $\mathrm{VD}$, a rat model of permanent bilateral common carotid artery occlusion is commonly used in experimental conditions since the two-vessel occlusion (2VO) method lowers cerebral blood flow significantly $(3,4)$. Despite its long term and mild characteristics, deprivation of oxygen and glucose in brain tissue causes a serious alterations, which are involved in the pathological changes and cognitive deficit, including generation of reactive oxygen species (ROS), neuroinflammation, mitochondrial dysfunction, neuronal apoptosis,

Bulent Ecevit University Faculty of Medicine, Department of Physiology, Zonguldak, Turkey

Address for correspondence: H.S. Ozacmak, Bulent Ecevit University Faculty of Medicine, Department of Physiology, Zonguldak, Turkey. Phone: +90.372.2613140, Fax: +90.372.2610264

Acknowledgement: This study was financially supported by the Bülent Ecevit University (Grant Number: 2014-26259946-01).
$\mathrm{A} \beta$ accumulation, synaptic dysfunction, white matter lesion, and tau hyperphosphorylation (1-6).

Previous studies demonstrated that $\mathrm{CCH}$ could activate the inflammatory glial cells (7-9), leading to various inflammatory reactions (9), ending up with cognitive impairment $(2,10) . \mathrm{CCH}$ can cause mitochondrial dysfunction and inhibition of protein synthesis, which in turn may change the balance of anti-oxidases and ROS towards to the accumulation of ROS producing oxidative damage $(11,12)$. In fact, many brain disorders including cognitive deficit are closely related to oxidative damage, which are usually involved in aging and AD (13).

In physiopathological onset of $\mathrm{CCH}$-induced damage, activated immune cells including microglia and astrocytes, chemokines, inflammatory pathways, and inflammatory mediators play crucial roles. In addition to oxidative stress, ischemic injury is enhanced with elevated generation of proinflammatory cytokines including interleukin-1 $\beta$ (IL-1 $\beta$ ), IL-6, and tumour necrosis factor- $\alpha$ (TNF- $\alpha$ ) during $\mathrm{CCH}(2)$.

Apoptosis is considered to be the major cell death mechanism occurring in $\mathrm{CCH}(1,14)$. Caspases, a family of thiol proteases, are dominant regulators of the apoptotic cascade. Ischemic insult to brain activates neuronal caspases. In particular, the major target associated with apoptosis is neuron. Apoptosis is induced by the ROS-mediated ischemia, resulting in disruption of blood brain bar- 
rier, inflammation and oxidative stress that eventually contribute to the brain damage (15).

Ethyl pyruvate (EP), a stable lipophilic ester derivative of pyruvate, was d previously reported to be beneficial in various experimental models in diseases, such as: intestinal ischemia (16), myocardial ischemia and reperfusion injury $(17,18)$, sepsis $(19,20)$, intracerebral haemorrhage (21), traumatic brain injury (22) acute pancreatitis (23), and middle cerebral artery occlusion $(24,25)$. Accordingly, EP provides multiple pharmacological effects, such as: scavenging ROS (26), suppressing inflammation (21), preventing apoptosis $(18,27)$, and supporting ATP synthesis (28). Thus, all of these previously published studies come up with a common point that EP might be a potential agent for the prevention and treatment of brain injury. On the other hand, there is no published study exploring the possible effects of $\mathrm{EP}$ on $\mathrm{CCH}$. Therefore, the current study, using a widely accepted model of $\mathrm{CCH}$ in rats, was aimed to evaluate the possible therapeutic potential of EP on cognitive processes, oxidant status, cell death, and neuroprotection of rats subjected to permanent occlusion of bilateral common carotid artery.

\section{Methods}

\section{Animals}

For the experiment, adult male Wistar rats (250-300 g) were used and housed in individual cages in a room maintained at $22 \pm 2{ }^{\circ} \mathrm{C}$ and $45-55 \%$ relative humidity with a $12 \mathrm{~h}$ light-dark cycle. They had free access to water and food. All experimental procedures involved with the animals were approved by the Ethics Committee of the Bülent Ecevit University. Maximum care for humanely approach to animals was one of primary purposes.

\section{Surgical procedure}

$\mathrm{CCH}$ was induced by a modified method of Cecethi et al (4). Briefly, rats were anesthetized with Ketamine $(90 \mathrm{mg} / \mathrm{kg}$ ) and Xylazine $(10 \mathrm{mg} / \mathrm{kg})$ followed by a midline cervical incision performed to expose bilateral common carotid arteries, which were carefully separated from vagal nerves and vessels. The right common carotid artery was ligated first with $5 / 0$ silk suture followed by closing of the wound. One week later, a new incision was made on the same region in order to occlude the left carotid artery in the same fashion. For animals of the sham-operated control group, the same operation was carried out without the ligation. Using a heating lamp and blanket, the rectal temperature was maintained at 37 ${ }^{\circ} \mathrm{C}$ throughout the surgery. Duration of $\mathrm{CCH}$ lasted thirty days.

\section{Drug administration and experimental design}

Thirty animals were randomly and equally divided into groups of sham, $\mathrm{CCH}$, and $\mathrm{CCH}$ treated with EP (Sigma, $50 \mathrm{mg} / \mathrm{kg} /$ day, i.p, for 30 days). The maximal brain protection was reached by the treatment of EP is the dose of $50 \mathrm{mg} / \mathrm{kg}$ which was consistent with the efficacious dose $(40-50 \mathrm{mg} / \mathrm{kg})$ determined in an adult rat cerebral ischemia model of MCAO (Shen et al, 2010) and other preclinical studies in rodents. Thus, EP was given intraperitoneally at the dose of $50 \mathrm{mg} / \mathrm{kg}$, beginning from the first day of the surgery and continuing through 30 days. Sham operated control animals were given only the physiological saline.

\section{Morris Water Maze Test}

Morris water maze test was performed as described previously (29). Briefly, the apparatus consisted of a circular water tank $120 \mathrm{~cm}$ in diameter and $60 \mathrm{~cm}$ in height, filled to a depth of $30 \mathrm{~cm}$ with opaque water and maintained at $25 \pm 1{ }^{\circ} \mathrm{C}$ of temperature. A hidden circular platform (10 cm in diameter) was submerged roughly $1.5 \mathrm{~cm}$ below the surface of the water and placed at the same location throughout the training period. Visual cues were fixed on to the walls of room. The tank was divided into four quadrants called north, east, south and west at equal distances on the rim. The platform was located in the centre of the northeast quadrant during training. Rats were given two trials per day to find the hidden platform for five consecutive days (maximum trial duration $60 \mathrm{~s}, 20 \mathrm{~s}$ reinforcement on the platform) during the training period of the task. The experimenter conducting the Morris water maze was blinded to the treatment groups. The rats were gently placed into the water, facing the side walls of the maze from one of the four starting position. In each trial, rats were given $60 \mathrm{~s}$ to swim in the pool to search for the platform. The latency to escape onto the hidden platform was measured. If the rat failed to find the platform within $60 \mathrm{~s}$, the training was terminated and a maximum score of $60 \mathrm{~s}$ was assigned. The rat was than guided to the hidden platform by hand, and it was allowed to stay on the platform for $20 \mathrm{~s}$.

\section{$M D A$ and $G S H$}

Oxidant and antioxidant status of rat brain was evaluated by measuring the tissue lipid peroxidation and reduced glutathione (GSH). For lipid peroxidation, the tissue content of malondialdehyde (MDA), a by-product of lipid peroxidation, was measured. Briefly, using a motor-driven pestle, tissue samples were homogenized in ice-cold trichloroacetic acid (TCA) by adding $10 \mathrm{ml}$ of $10 \%$ TCA per $g$ of tissue. After centrifugation, $750 \mu$ l supernatant was added to an equal volume of $0.67 \%$ thiobarbituric acid and heated to $100{ }^{\circ} \mathrm{C}$ for $15 \mathrm{~min}$. The absorbance of the samples was then measured spectrophotometrically at $535 \mathrm{~nm}$ (30). For measuring the GSH content of the samples, to the $0.5 \mathrm{ml}$ of supernatant obtained by using the same homogenization procedure as described above, $2 \mathrm{ml}$ of $0.3 \mathrm{M} \mathrm{Na}_{2} \mathrm{HPO}_{4}$ solution was added. A $0.2 \mathrm{ml}$ solution of dithiobisnitrobenzoate was added into the mixture, and the absorbance at $412 \mathrm{~nm}$ was measured immediately after vortexing (31).

\section{Caspase 3 and Na-K ATPase a subunit}

Enzyme linked immunosorbent assays (ELISA) for Caspase 3 (Cusabio Biotech Co., Ltd, Wuhan, China, Cat \#: CSB- CSBE08857r) and Na-K ATPase $\alpha$ subunit (Cusabio Biotech Co., Ltd, Wuhan, China, Cat \#: CSB-EL002322RA) were performed, according to the manufacturer's instructions.

\section{Plasma $I L-1 \beta$}

Plasma IL-1 $\beta$ level was measured using a commercial ELISA assay kit (Cusabio Biotech Co., Ltd, Wuhan, China, Cat \#: CSB- 


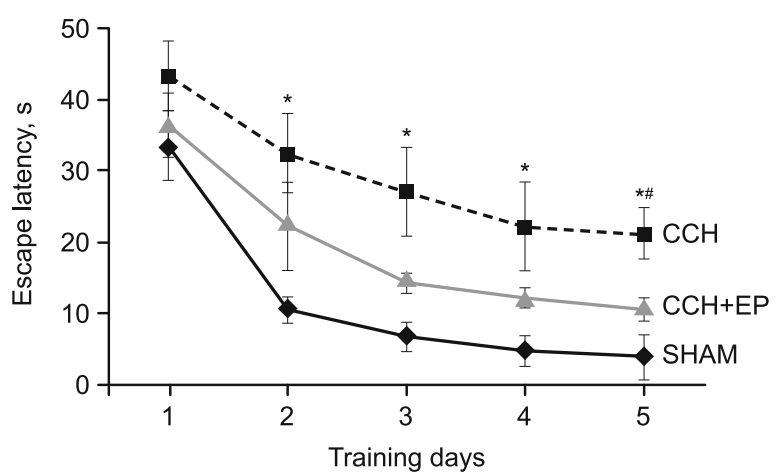

Fig. 1. Effect of EP on CCH-induced deficit in spatial learning and memory, which is determined using the MWM. The average escape latencies from different groups during training days. All values were expressed as the mean \pm the SEM $(n=10) .{ }^{*} p<0.05$ compared to the sham control; \# $\mathbf{p}<0.05$ compared to EP-treated group.

E08055r). The related procedure was carried out entirely in accordance with the kit's manual.

\section{Statistical analysis}

Each data point represents the mean \pm S.E.M. $(n=10$ for each group in all experiments). For statistical evaluation, SPSS 11.0 statistical software package program was used (SPSS Inc., Chicago, IL, USA). One-way analysis of variance (ANOVA) was applied for statistical comparison of the groups, followed by analysis with post hoc Bonferroni test to determine differences between the groups. Probability values of 0.05 or less were considered statistically significant.

\section{Results}

EP prevents the learning and memory impairment induced by $\mathrm{CCH}$

Learning and memory retention of Morris water maze were used to evaluate the spatial memory in rats. As shown in Figure 1, the average escape latency for the sham group decreased gradually throughout the five consecutive days. However, saline-treated CCH rats had longer escape latencies throughout the training days than sham-operated rats $(\mathrm{p}<0.05)$, demonstrating that hypoperfusion caused an obvious learning deficit in the model of $\mathrm{CCH}$. Following EP treatment $(50 \mathrm{mg} / \mathrm{kg})$ for 4 weeks, the average escape latency was significantly shorter on 5 th day of the test than that of salinetreated CCH group $(\mathrm{p}<0.05)$ (Fig. 1).

\section{EP suppresses oxidative stress}

To evaluate the effect of EP on $\mathrm{CCH}$-induced oxidative stress, the tissue contents of MDA and GSH were measured. The content of MDA in $\mathrm{CCH}$ group was significantly higher than that in the sham group $(p<0.05)$. After treatment with EP for 4 weeks, the content of MDA in EP-treated groups was markedly lower than that in the $\mathrm{CCH}$ group $(\mathrm{p}<0.05)$ (Fig. 2). However, the contents of GSH in both the $\mathrm{CCH}$ group and the treated group was not statistically different (Fig. 2) ( $p>0.05)$.

Effects on Na-KATPase a subunit level in hypoperfused brain

The effects of $\mathrm{CCH}$ and EP treatment on the expression of Na-K ATPase $\alpha$ subunit are shown on the Figure 3. In comparison to $\mathrm{CCH}$ and sham control groups, the treatment with EP was not significantly different in terms of the Na-K ATPase $\alpha$ subunit level. Another word, the treatment did not induce any alteration in Na-K ATPase $\alpha$ subunit levels in brain tissue.

\section{EP administration reduced the levels of caspase 3}

ELISA analysis revealed that the caspase 3 level was significantly higher in the $\mathrm{CCH}$ group than that in the sham group $(p<0.05)$ (Fig. 3). On the other hand, caspase 3 level was found to be significantly lower in the EP-treated $\mathrm{CCH}$ group compared to that in the $\mathrm{CCH}$ group $(\mathrm{p}<0.05)$ (Fig. 3).

\section{EP decreased plasma IL-1 $\beta$ level}

As demonstrated in Fig.3, $\mathrm{CCH}$ induced an elevation on the expression of the pro-inflammatory cytokine, IL-1 $\beta$, in comparison to the sham control. The treatment with EP, on the other hand,
A

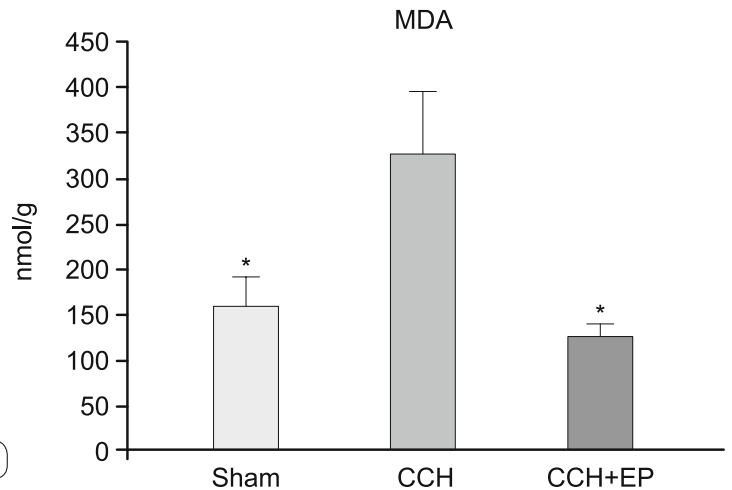

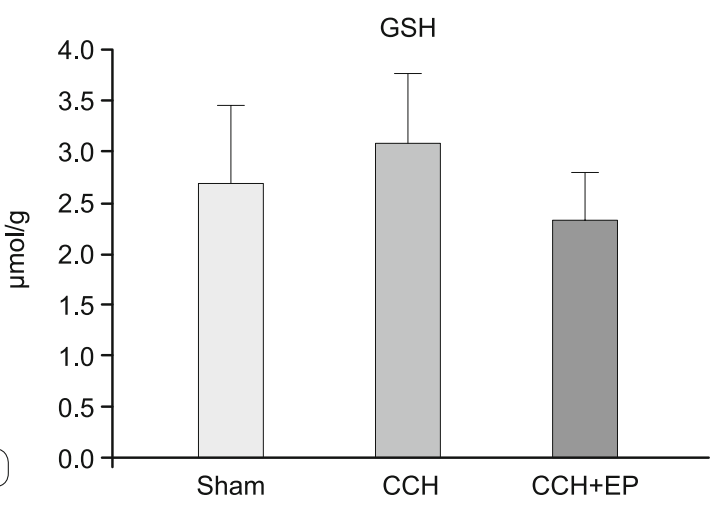

Fig. 2. Effect of EP treatment on (A) MDA and (B) GSH levels in brain tissue of hypoperfused rats. Values are expressed as the mean \pm S.E.M. $(\mathrm{n}=10) * \mathbf{p}<0.05$ compared to the $\mathrm{CCH}$ group. 

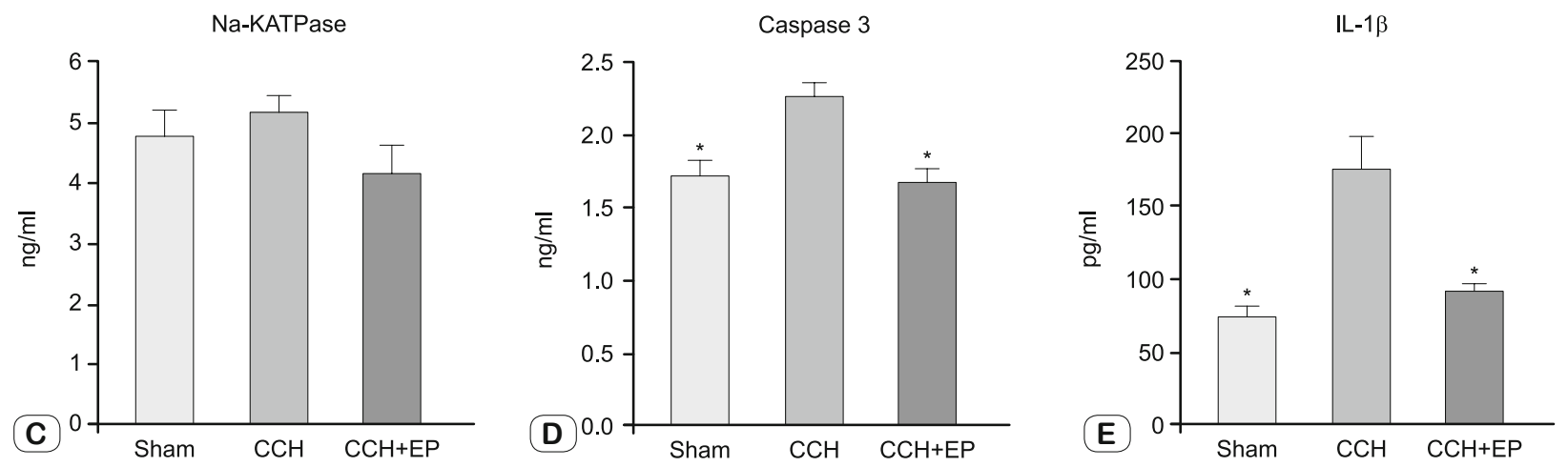

Fig. 3. Effect of EP treatment on (C) Na-K ATPase $\alpha$ subunit and (D) caspase 3 levels brain tissue and (E) IL-1 $\beta$ level in plasma of sham, CCH, and EP-treated groups. Values are expressed as mean \pm S.E.M. $(n=10) * p<0.05$ compared to $\mathrm{CCH}$ group.

decreased the IL-1 $\beta$ levels significantly in the hypoperfused brain, when compared with hypoperfused vehicle rats $(\mathrm{p}<0.05)$.

\section{Discussion}

$\mathrm{CCH}$ causes neurological damage in a long period of time that eventually ends up with a progressive cognitive and neurological dysfunction $(2,3)$. It is related to persistent decrease in cerebral blood flow (CBF) due to neurovascular dysfunction, consequently leading to cerebral ischemia (32). The current study demonstrated not only CCH-induced mechanism of neurodegeneration in part, but also the neuroprotective potential of EP for $\mathrm{CCH}$ type of ischemic injury.

In the present study, we showed that EP provided a remarkable attenuation of cognitive deficit triggered by $\mathrm{CCH}$. The possible mechanism of this beneficial effect might be associated with the followings: 1) the treatment with EP markedly reduced the oxidative stress; 2) the treatment with EP significantly decreased the tissue level of caspase 3; and 3) EP markedly lowered the level of proinflammatory cytokine IL- $1 \beta$ in plasma.

It is commonly known that $\mathrm{CCH}$ eventually results in neuronal death in such vulnerable region of the brain as hippocampus, which is especially associated with spatial learning and memory. Previously published studies demonstrated significant reductions in the number of pyramidal cells of the hippocampal CA1 region following $\mathrm{CCH}$, and therefore suggested that $\mathrm{CCH}$-induced cognitive impairment could possibly be associated with the loss of these cells $(14,29,33)$. Nevertheless, the mechanism responsible for $\mathrm{CCH}$ induced cognitive deficit appears to be very complex. Neuronal apoptosis of the hippocampal cells is related to cognitive deficit of rats subjected to $\mathrm{CCH}$ (34-36). Oxidative stress, mitochondrial dysfunction, and $\mathrm{Ca}^{2+}$ overload are all involved with the basic molecular and biological processes that end up with apoptosis (37). The principal effector caspase in the apoptotic cascade is caspase 3 (35). In general, the apoptotic signal first activates the apoptotic promoter caspase- 9 followed by the activation of apoptotic effector caspase 3 , leading to protein degradation and apoptosis. Caspase 3 activity, a common action point of several apoptotic pathways, is necessary for cascade reactions. Therefore, Caspase 3 is regarded to be a final apoptotic executor (38). Following $\mathrm{CCH}$, proapoptotic proteins like caspase 3 undergoes upregulation, which is one of the major causes of neuronal death in CCH-triggered injury (1). In the present study, $\mathrm{CCH}$ increased caspase 3 level and escape latencies in training sessions. Chronic treatment with EP significantly improved the impaired learning and memory abilities as indicated by markedly shorter escape latencies in the training sessions as well as lower caspase 3 levels. Moreover, the treatment normalized the generation of lipid peroxidation products in brain tissue subjected to $\mathrm{CCH}$. The oxidative damage due to $\mathrm{CCH}$ possibly plays an important role in neuronal apoptosis $(14,39)$, which is closely associated with the cognitive deficit observed in $\mathrm{CCH}$ (34). Our data suggests that EP may effectively attenuate the pro-apoptotic signals in the brain during $\mathrm{CCH}$. Thus, we can postulate that the anti-apoptotic property of EP might contribute to its neuroprotective effect. In fact, the anti-apoptotic effect of EP has recently been reported in cell culture and some animal models of neurological diseases $(27,40,41)$ and associated with alleviation of oxidative stress, up-regulation of $\mathrm{Bcl}-2$, and down-regulation of caspase 3 and Bax proteins $(27,42)$. However, the anti-apoptotic effect of EP in $\mathrm{CCH}$ has not been reported unless otherwise indicated. Here, we showed that the treatment with EP markedly reduced the caspase 3 level, oxidative stress, and cognitive impairment induced by $\mathrm{CCH}$. These finding are in line with previous studies showing that EP blocks caspase 3 activity in hypoxic brain injury (40).

The other mechanisms accounted for $\mathrm{CCH}$-induced cognitive impairment and cell death are related to generation of ROS and inflammation $(4,8,9,33,39,43,44)$. ROS and inflammatory glial cells are directly injurious to neurons; therefore, a substantial damage to the central nervous system takes place $(11,45)$. In particular, oxidative stress, changing the mitochondrial potential and inducing the release of the apoptosis-inducing factors, triggers a cascade of enzyme activities, which eventually causes the cell death by necrosis or apoptosis (14). ROS and lipid peroxidation may play a crucial role for the development of central nervous system lesions during CCH (39). MDA, a product of lipid peroxidation, is one of the oxidative stress markers. In the present study, 
$\mathrm{CCH}$ significantly elevated the tissue MDA content. An excessive production of ROS results in lipid peroxidation of the cell membranes; thus, subsequent damage is reflected by the accumulation of MDA (44). The related data appear to be consistent with those of the other studies reporting that the treatment with EP attenuates the lipid peroxidation $(18,28,46)$. The antioxidant effect of EP can occur mostly through its rapid and stoichiometrical scavenging effect on hydrogen peroxide (47).

It was previously reported that $\mathrm{CCH}$ initiates a significant microglial activation and neuroinflammatory responses in various regions of the rat brain, including the hippocampus $(7,33$, 48, 49). Moreover, it has been demonstrated that $\mathrm{CCH}$-induced neuroinflammation elevates the expression of pro-inflammatory cytokines, including TNF- $\alpha$ and IL- $1 \beta$, which play significant roles for progression of post-ischemic brain injury (50). Furthermore, activated microglia release pro-inflammatory cytokines, including TNF- $\alpha$ and IL-1 $\beta$, in the central nervous system (33). ROS are able to induce pro-inflammatory gene transcription in the microglia and astrocytes, causing various inflammatory reactions (9). Our findings showed that $\mathrm{CCH}$ resulted in elevated plasma levels of IL-1 $\beta$ in hypoperfused animals. The treatment with EP, however, decreased the plasma levels of the inflammatory cytokine. This result is consistent with the previous reports showing that EP has a significant anti-inflammatory activity. It has been recently reported that EP inhibited activation of microglia and inflammatory response in the post-ischemic brain $(25$, $51)$ and experimental intracerebral haemorrhage $(21,27)$. It does this by inhibiting NF- $\mathrm{B}$ activation and subsequent release of inflammatory mediators. NF- $\mathrm{kB}$ as a transcriptional activator in inflammatory responses, regulates the expression of many downstream inflammatory cytokines including TNF- $\alpha$ and IL-1 $\beta$ (21). EP has been shown to exert anti-inflammatory effect by inhibiting NF- $\mathrm{KB}$-dependent-pro-inflammatory signalling pathway in many disease models $(21,40,46)$. Inhibition of the DNA binding of $p 65$ (a nuclear factor [NF]- $\mathrm{KB}$ subunit) by EP via decreasing intracellular GSH level has been reported (52), resulting in the alteration of intracellular redox conditions to favour the oxidation of the key cysteine residue in $\mathrm{p} 65$. It has been reported that the anti-inflammatory effect of EP was attributable to the inhibition of ROS-dependent signal transducer and activator of transcription (STAT) signalling (51). In addition, it was recently reported that EP inducds p300 sequestration by Nrf2-suppressed p65 activation (53), which suggests a link between the anti-inflammatory and antioxidative functions of EP.

GSH plays a major role for defending cells against an oxidative insult. While glutathione peroxidase catalyses hydrogen peroxide into water to prevent hydroxyl radical formation, GSH becomes oxidized. In the present study, although the level of GSH appeared to be decreased in EP-treated group, there was no statistical difference among the groups. The anti-inflammatory effect of EP was reported to be, at least in part, by EP- induced depletion of cellular GSH stores (52). In the current study, decreased IL-1 $\beta$ levels in EP-treatment group might be related to this effect of EP.

One common feature observed in central nervous system pathologies involved with ischemic condition and TBI is the failure of the $\mathrm{Na}^{+}, \mathrm{K}^{+}$-ATPase pump because the pump is very vulnerable to oxidative stress $(54,55)$. Reduced activity can imply the membrane damage indirectly. Continuity of the enzymatic activity is "must" for the maintenance of normal neuronal function during the brain I/R insult (56). Some previously published studies showed that cerebral ischemia and some chronic neurodegenerative disorders led to decrease in $\mathrm{Na}^{+} \mathrm{K}^{+}$-ATPase activity (57). The $\mathrm{Na}^{+}$/ $\mathrm{K}^{+}$-ATPase is a heterodimer composed of an $\alpha$-catalytic subunit and a $\beta$-glycoprotein subunit. The $\alpha$ subunit has the binding site for the cations, ATP, and the inhibitor, ouabain. Thus, the most biologically active properties of the pump are commonly considered to be in its $\alpha$ subunit (58). In the present study, in comparison to $\mathrm{CCH}$ and sham control groups, the treatment with EP was not significantly different in terms of the Na-K ATPase $\alpha$ subunit level.

\section{Conclusion}

The present study is the first one to evaluate the effect of EP on rats subjected to $\mathrm{CCH}$. It was documented that the treatment with EP attenuated hypoperfusion-induced cognitive impairment. The potential mechanisms underlying for this attenuation were involved, at least, with alleviations of oxidative stress, neuroinflammation and apoptosis. Further studies that would focus on the effect of EP in depth are necessary for understanding the related mechanisms in detail. Thus, the results of the study strongly suggest the potential use of EP as a therapeutic agent for patients with vascular insufficiency in general and for those with cerebrovascular insufficiency in particular.

\section{References}

1. Stanojlović M, Guševac I, Grković I et al. Effects of chronic cerebral hypoperfusion and low-dose progesterone treatment on apoptotic processes, expression and subcellular localization of key elements within Akt and Erk signaling pathways in rat hippocampus. Neuroscience 2015; 311: $308-321$.

2. Du SQ, Wang XR, Xiao LY et al. Molecular Mechanisms of Vascular Dementia: What Can Be Learned from Animal Models of Chronic Cerebral Hypoperfusion? Mol Neurobiol 2016; 54 (5): 3670-3682.

3. Farkas E, Luiten PG, Bari F. Permanent, bilateral common carotid artery occlusion in the rat: A model for chronic cerebral hypoperfusionrelated neurodegenerative diseases. Brain Res Rev 2007; 54: 162-180.

4. Cechetti F, Pagnussat AS, Worm PV et al. Chronic brain hypoperfusion causes early glial activation and neuronal death, and subsequent longterm memory impairment. Brain Res Bull 2012; 87: 109-116.

5. Xi Y, Wang M, Zhang W et al. Neuronal damage, central cholinergic dysfunction and oxidative damage correlate with cognitive deficits in rats with chronic cerebral hypoperfusion. Neurobiol Learn Mem 2014; 109: 7-19.

6. Ueno Y, Koike M, Shimada Y et al. L-carnitine enhances axonal plasticity and improves white-matter lesions after chronic hypoperfusion in rat brain. J Cereb Blood Flow Metab 2015; 35: 382-391.

7. Farkas E, Donka G, de Vos RA, Mihály A, Bari F, Luiten PG. Experimental cerebral hypoperfusion induces white matter injury and microglial activation in the rat brain. Acta Neuropathol 2004; 108: 57-64. 
8. Wang J, Zhang HY, Tang XC. Huperzine a improves chronic inflammation and cognitive decline in rats with cerebral hypoperfusion. J Neurosci Res 2010; 88: 807-815.

9. Fu X, Zhang J, Guo L et al. Protective role of luteolin against cognitive dysfunction induced by chronic cerebral hypoperfusion in rats. Pharmacol Biochem Behav 2014; 26: 122-130.

10. Zhao Y, Gong CX. From chronic cerebral hypoperfusion to Alzheimer-like brain pathology and neurodegeneration. Cell Mol Neurobiol 2015; 35: $101-110$.

11. Zhang GL, Deng JP, Wang BH et al. Gypenosides improve cognitive impairment induced by chronic cerebral hypoperfusion in rats by suppressing oxidative stress and astrocytic activation. Behav Pharmacol 2011; 22: 633-644.

12. Orsucci D, Mancuso M, Ienco EC, Simoncini C, Siciliano G, Bonuccelli $\mathbf{U}$. Vascular factors and mitochondrial dysfunction: a central role in the pathogenesis of Alzheimer's disease. Curr Neurovasc Res 2013; 10: 76-80.

13. Manoharan S, Guillemin GJ, Abiramasundari RS, Essa MM, Akbar M, Akbar MD. The Role of Reactive Oxygen Species in the Pathogenesis of Alzheimer's Disease, Parkinson's Disease, and Huntington's Disease: A Mini Review. Oxid Med Cell Longev 2016: 8590578.

14. Ji HJ, Hu JF, Wang YH, Chen XY, Zhou R, Chen NH. Osthole improves chronic cerebral hypoperfusion induced cognitive deficits and neuronal damage in hippocampus. Eur J Pharmacol 2010; 636: 96-101.

15. Liu XL, Lu J, Xing J. Stabilization of HIF-1 $\alpha$ modulates VEGF and Caspase-3 in the hippocampus of rats following transient globalischemia induced by asphyxial cardiac arrest. Life Sci 2016; 151: 243-249.

16. Sims CA, Wattanasirichaigoon S, Menconi MJ, Ajami AM, Fink MP. Ringer's ethyl pyruvate solution ameliorates ischemia/reperfusion-induced intestinal mucosal injury in rats. Crit Care Med 2001; 29: 1513-1518.

17. Jang IS, Park MY, Shin IW, Sohn JT, Lee HK, Chung YK. Ethyl pyruvate has anti-inflammatory and delayed myocardial protective effects after regional ischemia/reperfusion injury. Yonsei Med J 2010; 51: 838-844.

18. Guo J, Zhang J, Luo $X$ et al. Effects of ethyl pyruvate on cardiac function recovery and apoptosis reduction after global cold ischemia and reperfusion. Exp Ther Med 2014; 7: 1197-1202.

19. Guarda IF, Correia CJ, Breithaupt-Faloppa AC et al. Effects of ethyl pyruvate on leukocyte-endothelial interactions in the mesenteric microcirculation during early sepsis treatment. Clinics (Sao Paulo) 2015; 70: 508-514.

20. Jiang Z, Li X, Lin Z, Chen J, Guan X, Chen M. Ethyl pyruvate reduces hepatic mitochondrial swelling and dysfunction in a rat model of sepsis. Int J Clin Exp Pathol 2015; 8: 7774-7785.

21. Su X, Wang H, Zhu L, Zhao J, Pan H, Ji X. Ethyl pyruvate ameliorates intracerebral hemorrhage-induced brain injury through anti-cell death and anti-inflammatory mechanisms. Neuroscience 2013; 245: 99-108.

22. Su X, Wang H, Zhao J, Pan H, Mao L. Beneficial effects of ethyl pyruvate through inhibiting high-mobility group box 1 expression and TLR4/NF-kappaB pathway after traumatic brain injury in the rat. Mediators Inflamm 2011: 1-10.

23. Luan ZG, Ma XC, Zhang H, Zhang C, Guo RX. Protective effect of ethyl pyruvate on pancreas injury in rats with severe acute pancreatitis. J Surg Res 2013; 181: 76-84.

24. Yu YM, Kim JB, Lee KW, Kim SY, Han PL, Lee JK. Inhibition of the cerebral ischemic injury by ethyl pyruvate with a wide therapeutic window. Stroke 2005; 36: 2238-2243.
25. Shin JH, Lee HK, Lee HB, Jin Y, Lee JK. Ethyl pyruvate inhibits HMGB1 phosphorylation and secretion in activated microglia and in the postischemic brain. Neurosci Lett 2014; 558: 159-163.

26. Liu J, Segal M, Yoo S et al. Antioxidant effect of ethyl pyruvate in respiring neonatal cerebrocortical slices after $\mathrm{H}(2) \mathrm{O}(2)$ stress. Neurochem Int 2009; 54: 106-110.

27. Fang R, Zheng X, Zhang M. Ethyl pyruvate alleviates early brain injury following subarachnoid hemorrhage in rats. Acta Neurochir (Wien) 2016; 158: 1069-1076.

28. Taylor MD, Grand TJ, Cohen JE et al. Ethyl pyruvate enhances ATP levels, reduces oxidative stress and preserves cardiac function in a rat model of off-pump coronary bypass. Heart Lung Circ 2005; 14: 25-31.

29. Sayan-Ozacmak H, Ozacmak VH, Barut F, Jakubowska-Dogru E. Neuroprotective efficacy of the peroxisome proliferator-activated receptor- $\gamma$ ligand in chronic cerebral hypoperfusion. Curr Neurovasc Res 2011; 8: 190-199.

30. Casini A, Ferrali M, Pampella A, Maellaro E, Combarti M. Lipid peroxidation and cellular damage in extrahepatic tissues of bromobenzene in toxicated mice. American Journal of Pathology 1989; 123: 520-531.

31. Aykac G, Uysal M, Yalcin AS, Kocak-Toker N, Sivas A, Oz H. The effects of chronic ethanol injection on hepatic lipid peroxide, glutathione, glutathione peroxidase and glutathione transferase in rats. Toxicology 1985; 36: 71-76.

32. Zhang X, Huang X, Fang Cun J, Li L. miR-124 Regulates the Expression of BACE1 in the Hippocampus Under Chronic Cerebral Hypoperfusion. Mol Neurobiol 2017; 54: 2498-2506.

33. Lee CH, Park JH, Ahn JH, Won MH. Effects of melatonin on cognitive impairment and hippocampal neuronal damage in a rat model of chronic cerebral hypoperfusion. Exp Ther Med 2016; 11: 2240-2246.

34. Bennett SA, Tenniswood M, Chen JH et al. Chronic cerebral hypoperfusion elicits neuronal apoptosis and behavioral impairment. Neuroreport 1998; 9: 161-166.

35. Jin W, Jia Y, Huang L et al. Lipoxin A4 methyl ester ameliorates cognitive deficits induced by chronic cerebral hypoperfusion through activating ERK/Nrf2 signaling pathway in rats. Pharmacol Biochem Behav 2014; 124: 145-152.

36. Yan ZQ, Chen J, Xing GX, Huang JG, Hou XH, Zhang Y. Salidroside prevents cognitive impairment induced by chronic cerebral hypoperfusion in rats. J Int Med Res 2015; 43: 402-411.

37. He Z, Liao Y, Zheng M, Zeng FD, Guo LJ. Piracetam improves cognitive deficits caused by chronic cerebral hypoperfusion in rats. Cell Mol Neurobiol 2008; 28: 613-627.

38. Min HM, Wang Y, Ren DY et al. Protective effect of 2-deoxy-Dglucose on the brain tissue in rat cerebral ischemia-reperfusion models by inhibiting Caspase-apoptotic pathway. Histol Histopathol 2017; 32: 57-67.

39. Sayan-Ozacmak H, Ozacmak VH, Barut F, Jakubowska-Dogru E. Rosiglitazone treatment reduces hippocampal neuronal damage possibly through alleviating oxidative stress in chronic cerebral hypoperfusion. Neurochem Int 2012; 61: 287-290.

40. Shen H, Hu X, Liu C et al. Ethyl pyruvate protects against hypoxicischemic brain injury via anti-cell death and anti-inflammatory mechanisms. Neurobiol Dis 2010; 37: 711722.

41. Jang M, Lee MJ, Cho IH. Ethyl pyruvate ameliorates 3-nitropropionic acid-induced striatal toxicity through anti-neuronal cell death and anti-inflammatory mechanisms. Brain Behav Immun 2014; 38: 151-165. 
42. Guo J, Zhang K, Ji Y, Jiang X, Zuo S. Effects of ethyl pyruvate on myocardial apoptosis and expression of Bcl-2 and Bax proteins after ischemia-reperfusion in rats. J Huazhong Univ Sci Technolog Med Sci 2008; 28: 281-283.

43. Huang L, He Z, Guo L, Wang H. Improvement of cognitive deficit and neuronal damage in rats with chronic cerebral ischemia via relative long-term inhibition of rho-kinase. Cell Mol Neurobiol 2008; 28: 757-768.

44. Ozacmak VH, Sayan H, Cetin A, Akyildiz-Igdem A. AT1 receptor blocker candesartan-induced attenuation of brain injury of rats subjected to chronic cerebral hypoperfusion. Neurochem Res 2007; 32: 1314-1321.

45. Annaházi A, Mracskó E, Süle $Z$ et al. Pre-treatment and post-treatment with alpha-tocopherol attenuates hippocampal neuronal damage in experimental cerebral hypoperfusion. Eur J Pharmacol 2007; 571: 120-128.

46. Wang P, Gong G, Wei Z, Li Y. Ethyl pyruvate prevents intestinal inflammatory response and oxidative stress in a rat model of extrahepatic cholestasis. J Surg Res 2010; 160: 228-235.

47. Atashfaraz E, Farokhi F, Najafi G. Protective effect of ethyl pyruvate on epididymal sperm characteristics, oxidative stress and testosterone level in methotrexate treated mice. J Reprod Infertil 2013; 14: 190-196.

48. Yang Z, Zhang N, Shen H, Lin C, Lin L, Yuan B. Microglial activation with reduction in autophagy limits white matter lesions and improves cognitive defects during cerebral hypoperfusion. Curr Neurovasc Res 2014; 11: 223-229.

49. Ma J, Zhang J, Hou WW et al. Early treatment of minocycline alleviates white matter and cognitive impairments after chronic cerebral hypoperfusion. Sci Rep 2015; 5: 12079.

50. Kim MS, Bang JH, Lee J, Han JS, Baik TG, Jeon WK. Ginkgo biloba L. extract protects against chronic cerebral hypoperfusion by modulating neuroinflammation and the cholinergic system. Phytomedicine 2016; 23: $1356-1364$.
51. Kim HS, Cho IH, Kim JE et al. Ethyl pyruvate has an anti-inflammatory effect by inhibiting ROS-dependent STAT signaling in activated microglia. Free Radic Biol Med 2008; 45: 950-963.

52. Song M, Kellum JA, Kaldas H, Fink MP. Evidence that glutathione depletion is a mechanism responsible for the anti-inflammatory effects of ethyl pyruvate in cultured lipopolysaccharide-stimulated RAW 264.7 cells. J Pharmacol Exp Ther 2004; 308: 307-316.

53. Kim SW, Lee HK, Shin JH, Lee JK. Up-down regulation of HO-1 and iNOS gene expressions by ethyl pyruvate via recruiting p300 to Nrf2 and depriving It from p65. Free Radic Biol Med 2013; 65: 468-476.

54. Lima FD, Souza MA, Furian AF et al. $\mathrm{Na}^{+}, \mathrm{K}+-\mathrm{ATP} a s e$ activity impairment after experimental traumatic brain injury: relationship to spatial learning deficits and oxidative stress. Behav Brain Res 2008; 193: 306-310.

55. Vaibhav K, Shrivastava $P$, Tabassum $R$ et al. Delayed administration of zingerone mitigates the behavioral and histological alteration via repression of oxidative stress and intrinsic programmed cell death in focal transient ischemic rats. Pharmacol Biochem Behav 2013; 113: 53-62.

56. Chen CM, Liu SH, Lin-Shiau SY. Honokiol, a neuroprotectant against mouse cerebral ischaemia, mediated by preserving $\mathrm{Na}+, \mathrm{K}+$-ATPase activity and mitochondrial functions. Basic Clin Pharmacol Toxicol 2007; 101: 108-116.

57. Simão F, Matté A, Matté $C$ et al. Resveratrol prevents oxidative stress and inhibition of $\mathrm{Na}(+) \mathrm{K}(+)$-ATPase activity induced by transient global cerebral ischemia in rats. J Nutr Biochem 2011; 22: 921-928.

58. Zhan L, Peng W, Sun W, Xu E. Hypoxic preconditioning induces neuroprotection against transient global ischemia in adult rats via preserving the activity of $\mathrm{Na}(+) / \mathrm{K}(+)$-ATPase. Neurochem Int 2011; 59: 65-72.

Received March 20, 2018. Accepted April 16, 2018. 